\title{
Avaliação geoestística de metais potencialmente tóxicos em solos urbanos, Paraná, Brasil
}

\begin{abstract}
Os elementos traços são poluentes comuns nos solos urbanos, tornando importante a avaliação dos mesmos. O objetivo principal deste estudo foi investigar a concentração de elementos traços em solos urbanos na região industrial de Curitiba, no Estado do Paraná, e analisar a aplicabilidade da modelagem geoestatística para a avaliação do impacto ambiental gerado. Para a análise dos elementos traços, foram coletadas amostras de solo até uma profundidade de $20 \mathrm{~cm}$ e submetidas a uma digestão ácida, de acordo com o método 3050B. A identificação dos elementos traços foi realizada através da técnica de Espectroscopia de Emissão Óptica. Foi também analisada a textura do solo e os dados foram tratados com a técnica de análise composicional. Para o mapeamento geoestatístico, foi utilizado o software R com o pacote geoR. A variabilidade espacial dos dados amostrados foi analisada previamente, calculando os indicadores estatísticos clássicos. Os resultados indicaram uma distribuição aproximadamente normal do conjunto de dados, o que é desejável. Em seguida, o estimador clássico de Matheron foi usado para calcular o semivariograma experimental para várias direções. Nenhum efeito de anisotropia foi mostrado. 0 semivariograma foi modelado a três modelos teóricos: esférico, exponencial e gaussiano. Os resultados da validação cruzada do modelo esférico sugeriram que ele seria o melhor modelo entre os três testados. Finalmente, as estimativas dos metais em locais desconhecidos foram obtidas por krigagem ordinária. Em relação aos valores obtidos de metais e os padrões estabelecidos por normativa específica sobre qualidade dos solos, apenas um local teve concentrações de $\mathrm{Ni}$ acima do permitido, estando os demais metais adequados. O mapa gerado mostrou claramente a variação espacial dos elementos traços na área estudada. Os resultados são adequados e sugerem que o uso desta metodologia fornece contribuições significativas para a gestão de áreas contaminadas por diferentes elementos traço.
\end{abstract}

Palavras-chave: Avaliação do Impacto Ambiental; Monitoramento; Solos; Inferência Espacial; Elementos Traço.

\section{Geostatistical assessment of trace metals in urban soils, Paraná, Brazil}

\begin{abstract}
Trace metals are common pollutants in urban soils thus making important the assessment of these pollutants. The main purpose of this study was investigated the concentrations of trace metals in urban soils in the industrial region of Curitiba, in the State of Paraná, Brazil and to examine the applicability of geostatistical modeling for assessing the environmental impact generated. For the analyses of trace elements, soil samples were collected at a depth of $0-20 \mathrm{~cm}$ and subjected to an acid digestion, in accordance with the 3050B method. The identification of trace elements was through the technique Spectroscopy of Optical Emission. The soil texture was also analyzed and the data were determined by the compositional analysis method. For the geostatistical mapping, it was used the software $\mathrm{R}$, with the geoR package. The spatial variability of the sampled data was analyzed previously calculating the classic statistical indicators. The results indicated an approximated normal distribution of the data samples which is desirable. Then, the Matheron's classical estimator was used to compute the experimental semivariogram for several directions. No effect of anisotropy was shown. The semivariogram was fitted to three theoretical models: spherical, exponential and Gaussian. The results of cross-validation of spherical model suggested that it is the best model among the others. Finally, the predictions of metals at unknown locations were obtained by ordinary kriging. In relation to the values obtained of metals and the standard values of the Brazilian referee legislation of management of contaminated sites, only one site had Ni levels higher than is allowed and other metals were ok. The generated map shows clearly the spatial variation of trace metals in the studied area. The results are suitable and suggest that the use of this methodology provides significant contributions to management of contaminated areas by different trace metals.
\end{abstract}

Keywords: Environmental Impact Assessment; Monitoring; Soils; Spatial Inference; Trace Elements.

Topic: Engenharia Ambiental

Reviewed anonymously in the process of blind peer.

Maurici Luzia Charnevski Del Monego (iD)

Universidade Tecnológica Federal do Paraná, Brasil http://lattes.cnpq.br/1410424302920222 http://orcid.org/0000-0002-9093-3802 maurici@utfpr.edu.br

Willian Hoffmann Hoffmann (iD)

Universidade Tecnológica Federal do Paraná, Brasil http://lattes.cnpq.br/3609845187064461

http://orcid.org/0000-0001-5283-542X

william dib@hotmail.com

Janaína de Fátima Gonzalez Munster Cicarello (iD) Universidade Tecnológica Federal do Paraná, Brasil http://lattes.cnpq.br/1372306361544865

http://orcid.org/0000-0003-1177-2244 janinha.cicarello@gmail.com

OI: 10.6008/CBPC2179-6858.2020.005.0032
Received: 05/08/2020 Approved: 27/09/2020

Larissa Kummer (iD

Universidade Tecnológica Federal do Paraná, Brasil

http://lattes.cnpq.br/7892203021390255

http://orcid.org/0000-0002-8282-2045

Ikummer@utfpr.edu.br

\section{Referencing this:}

MONEGO, M. L. C.; HOFFMANN, W. J.; CICARELLO, J. F. G. M.; KUMMER, L.. Avaliação geoestística de metais potencialmente tóxicos em solos urbanos, Paraná, Brasil. Revista Ibero Americana de Ciências Ambientais, v.11, n.5, p.339-351, 2020. DOI:

http://doi.org/10.6008/CBPC2179-6858.2020.005.0032 


\section{INTRODUÇÃO}

O bom entendimento do sistema solo é a chave para o sucesso individual e a harmonia ambiental de qualquer atividade humana que lida com a terra. A importância dos solos e dos sistemas pedológicos vem sendo cada vez mais reconhecida por líderes empresariais e políticos, pela comunidade científica e por todos aqueles que trabalham com a terra (BRADY, 2013).

O solo é o meio natural para o desenvolvimento das plantas terrestres, tal como se formou, ou modificado pelo ser humano. Este componente superficial é pertencente à costa terrestre, que está sob constante ação de agentes externos e internos que causam modificações naturais complexas. Existem fenômenos que afetam consideravelmente a configuração da superfície da Terra, como: flutuações de nível, tremores de terra, dobras e fraturas, vulcanismo e ações metamórficas com eles relacionadas, variação na profundidade, desagregação e transporte provocados pelo ar, água ou gelos. Assim, através destes intemperismos físicos e químicos, os produtos da desintegração da "rocha mãe", são arrastados e redistribuídos e novas porções da litosfera ficam ao alcance dos agentes atmosféricos. Concomitantemente, os sedimentos acumulados ficam sujeitos a novas alterações, que podem seguir novo ciclo de desagregação, transporte e depósito (COSTA, 2004).

Os elementos-traço são constituintes químicos negligenciáveis dos solos quantitativamente, mas são essenciais como micronutrientes para plantas. As primeiras publicações sobre elementos-traço foram dedicadas para problemas sobre a nutrição de plantas. Posteriormente, foi reconhecido que o comportamento de elementos-traço no solo varia amplamente, tanto para o elemento quanto para o solo, e que essas diferenças devem ser melhor compreendidas para a predição e gerenciamento da situação dos elementos-traço em solos. Mesmo que alguns desses elementos sejam, sobretudo, herdados das rochas formadoras, a sua distribuição nos perfis dos solos e sua divisão entre os componentes do solo refletem em vários processos pedogenéticos, assim como impactos causados por fatores externos, como por exemplo, práticas agrícolas, poluição, etc. (KABATA-PENDIAS et al., 2001).

O termo elemento-traço tem sido usado para definir metais catiônicos e oxiânicos presentes em baixas concentrações (usualmente $<1 \mathrm{~g} \mathrm{~kg}^{-1}$ ) em solos e plantas, muito embora Alumínio (Al), Ferro (Fe) e Titânio (Ti), os quais ocorrem em maiores concentrações na litosfera, também sejam definidos como elementos-traço (FREIRE, 2011).

Os elementos-traço, como o cobre, o cádmio, a prata, o arsênio, o cromo e o mercúrio, que são tóxicos a concentrações relativamente baixas, persistem no ambiente e podem se acumular em níveis que interrompem o crescimento das plantas e interferem na vida animal. Os detritos de atividades mineradoras e industriais e o lodo de esgoto são fontes de concentração de metais pesados potencialmente prejudiciais (KUMMER et al., 2013).

Para a detecção e quantificação dos elementos-traço, pode-se utilizar a técnica de espectroscopia de emissão usando Espectrometria de Emissão Óptica (ICP-OES). Este é um método rápido, sensível e conveniente para a determinação de elementos, incluindo metais em solução. Todas as matrizes, incluindo 
águas subterrâneas, amostras aquosas, resíduos sólidos, solos, lodos, sedimentos, dentre outros, necessitam de procedimentos de digestão das amostras antes de realizar as análises. A precisão e exatidão dos resultados obtidos utilizando este método são suficientes para a execução da maioria dos trabalhos analíticos (CSUROS et al., 2002).

As abordagens que têm sido utilizadas para a análise de dados ambientais, como a concentração de elementos-traço presentes em solos, não têm sido verdadeiramente esclarecedoras dos reais impactos sobre o ambiente (MONEGO et al., 2010). A metodologia geoestatística é uma técnica que está sendo utilizada com sucesso para a análise e caracterização da variabilidade espacial de propriedades do solo. Vem como uma alternativa para a resolução das dificuldades apresentadas, como elevados custos das campanhas de monitoramento, baixo volume de dados e tempo desprendido para a execução de todas as análises necessárias (SABY et al., 2006).

A metodologia geoestatística pode ser usada em diversas áreas e muitas pesquisas integraram com sucesso o método: na medição da dependência espacial da chuva e da erosividade para o estado do Ceará (RIBEIRO FILHO et al., 2017); para estimar a temperatura média anual do estado da Bahia (ALMEIDA et al., 2017); na variabilidade espacial do bário na área com resíduos de perfuração de poços de petróleo (SOBRINHO et al., 2018); na avaliação da precisão posicional absoluta dos dados geoespaciais (SANTOS, 2017). Outros trabalhos aplicaram métodos geoestatísticos para prever o gradiente de distribuição de possíveis contaminações por metais em várias partes do mundo (CHEN et al., 2008; GARCÍA-LORENZO et al., 2012; FINZGAR et al., 2014; NEZHAD et al., 2014). In one of his works, Lado et al. (2008) showed the application of geostatistics to model the distribution of eight trace elements $(\mathrm{As}, \mathrm{Cd}, \mathrm{Cr}, \mathrm{Cu}, \mathrm{Hg}, \mathrm{Ni}, \mathrm{Pb}$ and Zn). Lado et al. (2008) em um de seus trabalhos mostra a aplicação da geoestatística utilizada para modelar a distribuição de oito elementos-traço ( $\mathrm{As}, \mathrm{Cd}, \mathrm{Cr}, \mathrm{Cu}, \mathrm{Hg}, \mathrm{Ni}, \mathrm{Pb}$ e $\mathrm{Zn}$ ).

No município de Curitiba, estado do Paraná, de acordo com o Instituto de Pesquisa e Planejamento Urbano no município, a Regional CIC localiza-se na porção oeste, fazendo divisa com os municípios de Campo Largo e Araucária. A área total da Regional é de 6.003 hectares, o que corresponde a 13,81\% do território de Curitiba. Ela é composta por quatro bairros: Cidade Industrial de Curitiba - CIC, Augusta, São Miguel e Riviera. O CIC é o maior dos bairros, correspondendo a $68 \%$ da extensão territorial da Regional. Este bairro apresenta uma densidade populacional igual a 39,08 hab/ha, com cerca de 159.820 habitantes em 2010 (IPPUC, 2014).

A concepção de uma área de uso predominantemente industrial e sua efetivação em Curitiba se deu em 1973, quando a área escolhida foi declarada como de utilidade pública para fins de desapropriação, bem como foi formulada a proposta de implantação do que passou a ser denominada Cidade Industrial de Curitiba (CIC). Em 1975, ocorreram alterações no zoneamento, definindo a Cidade Industrial como local destinado essencialmente ao uso industrial; com isso, foram criados artifícios impeditivos para novas instalações em outras áreas da cidade (MOURA et al., 2009).

De acordo com os dados socioeconômicos da Agência Curitiba (2010), em 2004 esse bairro apresentava um número de 818 indústrias, sendo que no ano de 2010 houve um aumento para 1560, refletindo em um crescimento de $91 \%$. Além disso, este bairro contém estabelecimentos econômicos na 
administração voltados em $31,49 \%$ para prestação de serviços, $46,18 \%$ para o comércio e $21,62 \%$ para o setor industrial (CURITIBA, 2010). Sendo o CIC uma região que abriga muitas indústrias do município de Curitiba, Paraná, esta foi escolhida dentre os outros bairros para a análise dos teores de elementos-traço e realização dos trabalhos de mapeamento geoestatístico.

\section{MATERIAIS E MÉTODOS}

Inicialmente, foram coletadas 34 amostras georreferenciadas de solo do bairro $\mathrm{CIC}$ em Curitiba Paraná, a uma profundidade de $0-20 \mathrm{~cm}$ (Figura 1).

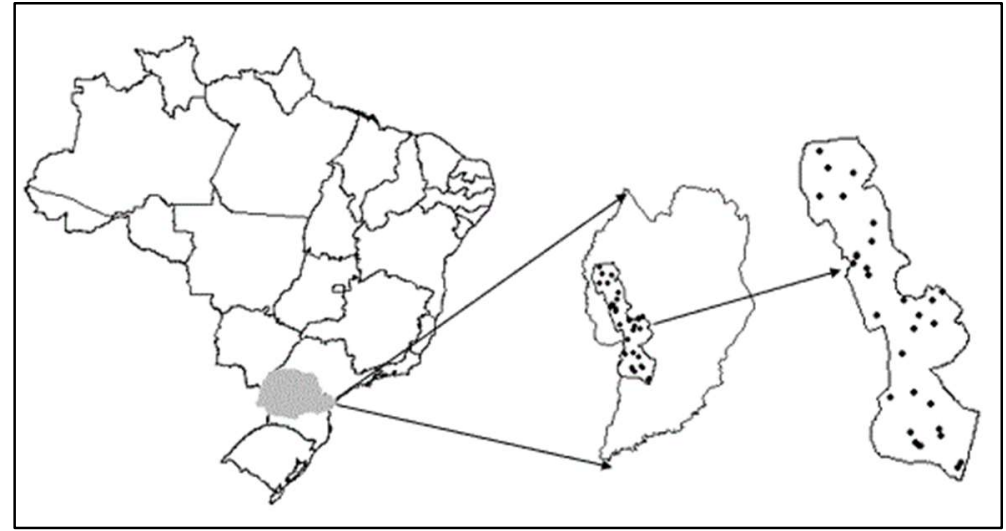

Figura 1: Localização da área de estudo.

As amostras de solo foram secas ao ar e peneiradas $(2 \mathrm{~mm})$ para obter terra fina seca ao ar (TFSA). Essas amostras foram analisadas no Laboratório de Análises de Solo da Universidade Tecnológica Federal do Paraná (UTFPR). A textura do solo foi determinada pelos métodos tradicionais de análise do solo (EMBRAPA, 1997). Para analisar os dados composicionais da granulometria, foi utilizado o pacote denominado "geoComp" desenvolvido por Martins (2010) e o pacote denominado "composições" desenvolvido por Pawlowsky-Glahn et al. (2004), que considera as variáveis espacialmente distribuídas. Optou-se por ser adequado para a análise de dados em proporções e que somam $100 \%$.

Para as análises de elementos-traço, as amostras de solo passaram pelo processo de digestão ácida, de acordo com o método 3050B de Digestão Ácida de Sedimentos, Lodos e Solos, da Agência Norte Americana de Proteção Ambiental (USEPA, 1996). Utilizando um ICP-OES foram analisados seis metais potencialmente tóxicos: $\mathrm{Cd}$ (cádmio), $\mathrm{Cr}$ (cromo), $\mathrm{Cu}$ (cobre), Ni (níquel), Pb (chumbo) e Zn (zinco).

Uma vez obtidos os teores dos elementos-traço, foram seguidas as principais etapas da metodologia geoestatística para a análise, como sugere a literatura, utilizando o software R (R DEVELOPMENT CORE TEAM) e o pacote geoR (RIBEIRO JUNIOR et al., 2001): i) Primeiramente foram realizadas análises exploratórias dos dados; ii) em seguida os semivariogramas experimentais foram construídos e modelados; iii) foram utilizados métodos de ajuste dos modelos dos semivariogramas e a técnica de validação cruzada para a validação dos modelos dos semivariogramas; iv) foi utilizado o método de krigagem ordinário para o cálculo das estimativas (ISAAKS et al., 1989; SOARES, 2000; CLARK et al., 2000; WACKERNAGEL, 2003).

A análise exploratória, como primeiro passo, consiste em fazer um diagnóstico do comportamento 
dos dados disponíveis. Foi realizada uma análise estatística descritiva e espacial para o conhecimento do comportamento dos dados, características da distribuição, entre outros. Ainda na mesma etapa, verificou-se a existência da dependência espacial das grandezas em estudo. Uma vez detectava a existência de dependência espacial entre os dados, esta foi quantificada através do semivariograma. Esta função corresponde à metade da variância, ou seja, a semivariância, das diferenças entre os valores observados com a distância de separação entre esses pontos. A interpretação do semivariograma fornece o grau de continuidade espacial e modo de dispersão da variável usada na previsão espacial. O estimador clássico de semivariância de Matheron foi utilizado neste estudo, representado através da equação (CRESSIE, 1993):

$$
\gamma(h)=\frac{1}{2 N(h)} \sum_{i=1}^{N(h)}\left[Z\left(x_{i}\right)-Z\left(x_{i}+h\right)\right]^{2}
$$

Onde $\gamma(h)$ é o semivariograma, $Z\left(x_{i}\right)$ é o valor da concentração medido (valor amostral) na localização $x_{i}, h$ é a distância entre as localizações e $N(h)$ é o número de pares de medidas distantes $h$ entre si (CRESSIE, 1993; KITANIDIS, 1997). O semivariograma experimental foi calculado para várias distâncias. Após a construção do semivariograma experimental, escolheu-se o modelo teórico dentre os modelos (esférico, exponencial e gaussiano) que melhor se ajustasse à conduta espacial dos dados. Para auxiliar na escolha do melhor modelo, foi realizada a técnica de validação cruzada. Essa técnica consiste em escolher o modelo que melhor represente o comportamento dos dados em função da menor estatística de erro. Nesse processo, cada uma das observações é temporariamente retirada do conjunto de dados e o seu valor é estimado utilizando as restantes, repetindo-se para todas as observações. Os valores estimados e os valores reais podem ser comparados (i) usando uma mesma análise estatística para esses dois conjuntos de dados e/ou (ii) efetuando uma análise estatística dos erros. Na análise estatística dos erros são habitualmente calculados os seguintes indicadores estatísticos: erro médio (ME); raiz quadrada do erro quadrático médio (RMSE), raiz quadrada da variância de krigagem média (RMKV), erro estandardizado médio (MSE) e raiz quadrada do erro estandardizado quadrático médio (RMSSE) dados, respectivamente pelas equações:

$$
\begin{aligned}
& M E=\frac{1}{N} \sum_{i=1}^{N}\left[\hat{Z}\left(x_{i}\right)-Z\left(x_{i}\right)\right] \\
& R M S E=\sqrt{\frac{1}{N} \sum_{i=1}^{N}\left[\hat{Z}\left(x_{i}\right)-Z\left(x_{i}\right)\right]^{2}} \\
& R M K V=\sqrt{\frac{1}{N} \sum_{i=1}^{N} \sigma^{2}\left(x_{i}\right)} \\
& M S E=\frac{1}{N} \sum_{i=1}^{N}\left[\frac{\hat{Z}\left(x_{i}\right)-Z\left(x_{i}\right)}{\sigma^{2}\left(x_{i}\right)}\right] \\
& R M S S E=\sqrt{\frac{1}{N} \sum_{i=1}^{N}\left[\frac{\hat{Z}\left(x_{i}\right)-Z\left(x_{i}\right)}{\sigma^{2}\left(x_{i}\right)}\right]^{2}}
\end{aligned}
$$


onde $\hat{Z}\left(x_{i}\right)$ é o valor estimado e $\mathrm{Z}\left(\mathrm{x}_{\mathrm{i}}\right)$ o valor observado na posição $\mathrm{x}_{\mathrm{i}} ; \mathrm{N}$ é o número de observações estimadas;

$e^{\sigma^{2}\left(x_{i}\right)}$ é a variância da estimativa para o ponto $x_{i}$ (ou variância do erro de estimação). Será desejável que ME seja próximo de zero, indicando que o estimador é não enviesado. O valor de REQM deve ser o menor possível, indicando que os valores das estimativas são próximos dos valores medidos. O valor de RVKM deve ser naturalmente o menor possível. Se as variâncias de krigagem forem precisas, então o valor de RESQM deve ser próximo de 1 (WACKERNAGEL, 2003).

Por fim, aplicou-se a interpolação por krigagem nos pontos não amostrados. Esse método de interpolação prevê não só um valor, mas também uma medida da incerteza associada a esse valor, havendo maior confiança nos dados estimados. Este valoriza a correlação dos dados em pequenas distâncias utilizando ponderadores que atribuem pesos maiores a observações que estão mais próximas. Ao final foi possível obter os mapas do gradiente de concentração para cada elemento-traço e a dispersão destes poluentes na região de estudo.

\section{RESULTADOS E DISCUSSÃO}

A composição granulométrica, que pode expressar propriedades importantes do solo, incluindo a classificação do solo, é a textura do solo, sendo expressa pela proporção relativa de areia, silte e argila exemplo típico da análise composicional (PEIXOTO et al., 2011; GRUNSKY, 2002). Os histogramas de frações de areia, silte e argila e o diagrama ternário (Figura 2) apresentam as composições amostradas. O silte foi o componente que teve o menor valor devido à distância dos pontos do vértice do diagrama. Neste diagrama, os pontos próximos ao vértice têm uma alta porcentagem desse componente e baixas proporções dos outros dois componentes. Existe uma proporção maior de argila e areia em relação ao silte. A análise composicional revela que as amostras de solo são predominantemente arenosas e argilosas. Essas características são importantes para avaliar o potencial de acúmulo de metais pesados no solo, uma vez que sua presença em solos arenosos é mais significativa, devido à possibilidade de lixiviação e alcance de águas subterrâneas.

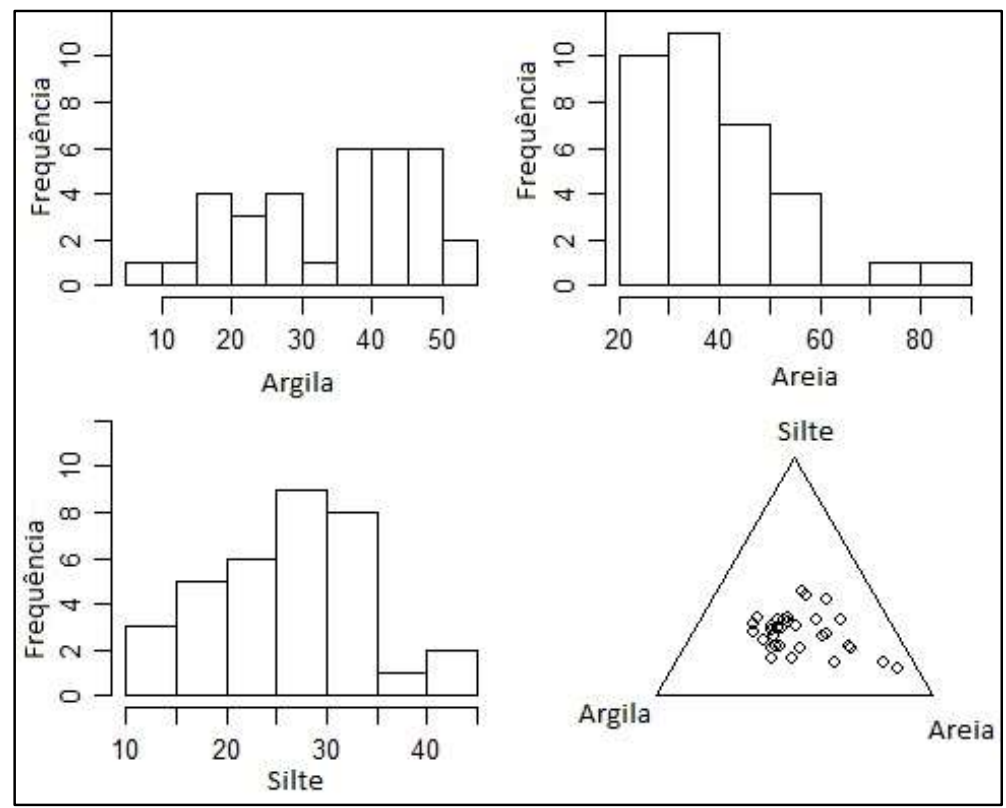

Figura 2: Distribuição de argila, areia, silte e o diagrama ternário.

Foram seguidas as etapas para a aplicação da metodologia geoestatística na análise dos seis metais: 
$\mathrm{Cd}, \mathrm{Cr}, \mathrm{Cu}, \mathrm{Ni}, \mathrm{Pb}$ e Zn. A Tabela 1 apresenta a análise estatística descritiva para a concentração do elemento $\mathrm{Cd}$, com o valor em $\mathrm{mg} \mathrm{kg}^{-1}$. Estes dados servem como base para identificar a possibilidade de realizar a interpolação e gerar o mapa geoestatístico.

Tabela 1: Estatística descritiva dos elementos - traço analisados $\left(\mathrm{mg}^{\mathrm{kg}} \mathrm{kg}^{-1}\right)$.

\begin{tabular}{lllllll}
\hline Sumário & $\mathbf{C d}$ & $\mathbf{P b}$ & $\mathbf{C u}$ & $\mathbf{C r}$ & $\mathbf{N i}$ & $\mathbf{Z n}$ \\
\hline Mínimo & 0.04 & 7.70 & 8.92 & 12.35 & 3.77 & 12.32 \\
Quartil inferior & 0.16 & 9.82 & 17.18 & 19.68 & 6.40 & 15.48 \\
Média & 0.19 & 12.85 & 20.50 & 25.28 & 10.24 & 26.39 \\
Quartil superior & 0.20 & 14.59 & 22.64 & 28.61 & 8.96 & 34.16 \\
Mediana & 0.18 & 11.78 & 19.13 & 25.26 & 7.28 & 24.32 \\
Máximo & 0.57 & 31.11 & 48.69 & 49.36 & 78.76 & 58.64 \\
Variância & 0.007 & 21.78 & 47.99 & 72.06 & 156.08 & 160.12 \\
Desvio padrão & 0.09 & 4.67 & 6.93 & 8.49 & 12.49 & 12.65 \\
Coeficiente de variação & 46.29 & 36.05 & 33.39 & 33.58 & 122.00 & 47.95 \\
Coeficiente de assimetria & 2.45 & 1.82 & 1.86 & 0.74 & 4.84 & 0.86 \\
Curtose & 9.15 & 4.62 & 5.73 & 0.47 & 23.63 & -0.21 \\
\hline
\end{tabular}

O coeficiente de assimetria obtido foi de 2,45 e coeficiente de curtose foi de 9,15 (curva normal tendendo a ser mais afunilada). Observando o mínimo $\left(0,04 \mathrm{mg} \mathrm{kg}^{-1}\right)$ e máximo $\left(0,57 \mathrm{mg} \mathrm{kg}^{-1}\right)$, verificou-se que o intervalo da amplitude é baixo, o que pode nos mostrar uma homogeneidade nos dados. Percebeu-se com isso um comportamento dos dados referente ao $\mathrm{Cd}$ próximo à normalidade, viabilizando a aplicação do método. Foram testados três modelos matemáticos para modelar os variogramas: o esférico, o exponencial e o gaussiano.

O gráfico de quartis, Figura 3 (I), mostra pontos próximos com valores similares, dando-nos evidências de que existe dependência espacial. Assim, parte-se para a quantificação da dependência espacial do fenômeno, que é feita através do variograma que pode ser observado na Figura 3 (II). A distância adotada, na qual foi observada correlação especial entre os dados, foi de $1500 \mathrm{~m}$. O modelo escolhido para gerar o mapa das estimativas foi o esférico, escolhido com o auxílio da análise na validação cruzada.



Figura 3: Da esquerda para a direita, os quartis do elemento $\mathrm{Cd}(\mathrm{I})$, semivariograma experimental omnidirecional e modelos ajustados de Cd (II) e o mapa de previsão de distribuição de Cd usando o modelo esférico (III).

A Figura 3 (III) apresenta o mapa das estimativas do elemento Cd. A coloração clara indica uma menor concentração (cerca de $0,1 \mathrm{mg} \mathrm{kg}^{-1}$ ) e a coloração mais escura, próxima a preto, indica concentrações mais elevadas (acima de 0,5 $\mathrm{mg} \mathrm{kg}^{-1}$ ). Observando o mapa das estimativas, pode-se perceber que na região central, mais à direita, encontram-se as maiores concentrações de $\mathrm{Cd}$ juntamente com um ponto mais isolado, ao sul. A Tabela 1 apresenta a análise estatística descritiva para a concentração do elemento $\mathrm{Pb}$, com valores em mg kg-1. O coeficiente de assimetria obtido foi de 1,87, e coeficiente de curtose com valor de 4,62 o qual se refere a uma curva mais larga. 
Observando o mínimo $\left(7,70 \mathrm{mg} \mathrm{kg}^{-1}\right)$ e máximo $\left(31,11 \mathrm{mg} \mathrm{kg}^{-1}\right)$, verificou-se que o intervalo da amplitude mostra novamente uma homogeneidade nos dados e comportamento próximo à normalidade. A distância adotada para o variograma foi de $340 \mathrm{~m}$. O gráfico de quartis (Figura 4 (I)) mostra pontos próximos com valores similares, confirmando a existência de dependência espacial. O variograma experimental e os modelos testados estão apresentados na Figura 4(II). Tanto o modelo esférico quanto o exponencial mostraram-se, a partir da análise de validação cruzada, possíveis de serem utilizados para gerar o mapa geoestatístico com parâmetros adequados.

O mapa foi gerado utilizando o modelo esférico (ver Figura 4 (III)). Esse mostra que na região de coloração clara há uma menor concentração (cerca de $10 \mathrm{mg} \mathrm{kg}^{-1}$ ) deste elemento e a coloração mais escura, próxima a preto, indica concentrações mais elevadas (acima de $30 \mathrm{mg} \mathrm{kg}^{-1}$ ). Neste mapa pode ser visualizado que na região central encontram-se os valores estimados de concentração de $\mathrm{Pb}$ mais altos.

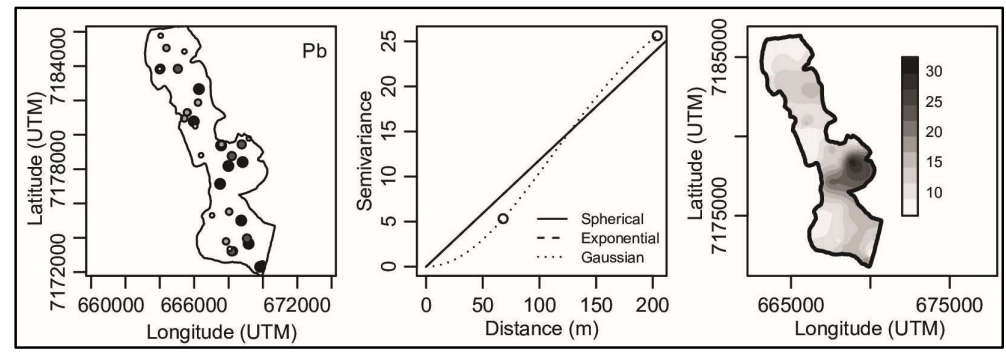

Figura 4: Da esquerda para a direita, os quartis do elemento $\mathrm{Pb}(\mathrm{I})$, semivariograma experimental omnidirecional e modelos ajustados de $\mathrm{Pb}$ (II) e mapas de previsão de distribuição de $\mathrm{Pb}$ usando o modelo esférico (III).

A Tabela 1 apresenta a estatística descritiva para a concentração do elemento $\mathrm{Cu}$, com valores em $\mathrm{mg} \mathrm{kg}^{-1}$. Verifica-se que há uma pequena assimetria semelhante ao metal anterior $(\mathrm{Pb})$ com coeficiente de assimetria de 1,86. O coeficiente de curtose, com valor de 5,73, o que indica uma curva normal um pouco afunilada. Observando o mínimo $\left(8,92 \mathrm{mg} \mathrm{kg}^{-1}\right)$ e máximo $\left(48,69 \mathrm{mg} \mathrm{kg}^{-1}\right)$, novamente verificou-se que o intervalo da amplitude mostra homogeneidade nos dados.

Da mesma maneira, o gráfico de quartis (ver Figura 5 (I)) apresenta evidências de que existe dependência espacial, onde pode ser verificado que quartis que representam valores mais baixos estão mais próximos e quartis de valores mais elevados também seguem essa tendência. A distância adotada, onde foi observada correlação especial entre os dados, foi de $90 \mathrm{~m}$. O modelo escolhido (exponencial) apresentou um range muito maior do que os outros modelos para interpolação do mapa e os valores mínimos e máximos mais próximos dos dados reais obtidos, verificado na Tabela 1. Esta escolha também foi confirmada pela validação cruzada.
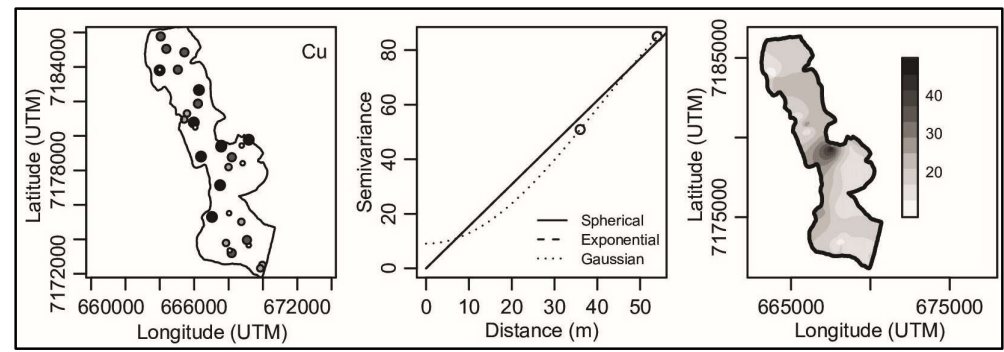

Figura 5: Da esquerda para a direita, os quartis do elemento $\mathrm{Cu}(\mathrm{I})$, semivariograma experimental omnidirecional e modelos ajustados de $\mathrm{Cu}$ (II) e mapas de previsão de distribuição de $\mathrm{Cu}$ usando o modelo exponencial (III). 
No mapa da Figura 5 (III) observa-se a variação de concentração de Cu estimada para esta região. A coloração clara indica uma menor concentração (inferiores a $20 \mathrm{mg} \mathrm{kg}^{-1)} \mathrm{e}$ a coloração mais escura, próxima ao preto indica concentrações mais elevadas (acima de $40 \mathrm{mg} \mathrm{kg}^{-1}$ ). A região com a coloração mais escura está localizada um pouco acima da central, uma área mais afunilada do mapa.

A Tabela 1 mostra a estatística descritiva para a concentração do elemento $\mathrm{Cr}$, com valores em $\mathrm{mg}$ $\mathrm{kg}^{-1}$. Verifica-se que há uma pequena assimetria na distribuição dos dados com coeficiente de assimetria de 0,74 , e coeficiente de curtose, com valor de 0,47 , e isso representa uma curva normal mais larga e de formato semelhante à gaussiana. Observando o mínimo $\left(12,35 \mathrm{mg} \mathrm{kg}^{-1}\right)$ e máximo $\left(49,36 \mathrm{mg} \mathrm{kg}^{-1}\right)$, verificou-se que o intervalo da amplitude é relativamente pequeno.

No gráfico do quartil, Fig. 6 (I), algumas evidências de dependência espacial. Para entender melhor a disposição dos dados para $\mathrm{Cr}$, foram observados valores das variâncias para todas as distâncias associadas, através de uma análise mais detalhada do variograma. Verificou-se que, em alguns casos, distâncias muito pequenas apresentaram uma grande variação associada e distâncias maiores apresentaram variações menores. A distância adotada para o semivariograma foi de $1000 \mathrm{~m}$. Os modelos testados para o semivariograma experimental são mostrados na Fig. 6 (II). O modelo esférico mostrou-se, de acordo com a análise de validação cruzada, adequado para gerar o mapa geoestatístico com parâmetros apropriados.

Na Figura 6 (III), a variação estimada na concentração de $\mathrm{Cr}$ para esta área. A cor clara indica concentrações mais baixas (menos de $20 \mathrm{mg} \mathrm{kg}^{-1}$ ) e a cor escura, quase preta, indica concentrações mais altas (acima de $40 \mathrm{mg} \mathrm{kg}^{-1}$ ). Observando o mapa das estimativas, as maiores concentrações de $\mathrm{Cr}$ podem ser vistas na região central, além de um ponto mais isolado no sul.

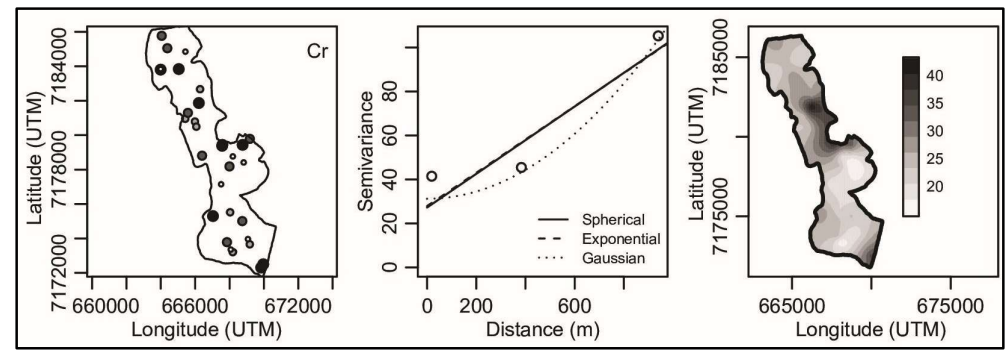

Figura 6: Da esquerda para a direita, os quartis do elemento $\mathrm{Cr}(\mathrm{I})$, semivariograma experimental omnidirecional e modelos ajustados de $\mathrm{Cr}$ (II) e mapas de previsão de distribuição de $\mathrm{Cr}$ usando o modelo esférico (III).

Na Tabela 1 apresenta-se a análise estatística para a concentração do elemento $\mathrm{Ni}$, com valores em $\mathrm{mg} \mathrm{kg}^{-1}$. Nota-se que há uma assimetria na distribuição dos dados com coeficiente de assimetria de 4,84, e coeficiente de curtose, com valor de 23,63, ou seja, uma curva normal bastante afunilada. Observando o mínimo $\left(3,77 \mathrm{mg} \mathrm{kg}^{-1}\right)$ e máximo $\left(78,76 \mathrm{mg} \mathrm{kg}^{-1}\right)$, verificou-se que o intervalo da amplitude é mais alto que os anteriores $(\mathrm{Cd}, \mathrm{Cu}$ e $\mathrm{Pb})$. Ainda assim, existe um comportamento próximo à normalidade, viabilizando a aplicação do interpolador.

O gráfico de quartis (Figura 7 (I)) nos dá, mais uma vez, evidências de que existe dependência espacial. A distância adotada, onde foi observada correlação especial entre os dados, foi de $1500 \mathrm{~m}$. 0 variograma experimental foi modelado (ver Figura 7 (II)) e modelo adotado para gerar o mapa de estimativas 
foi o esférico, com um range maior que os demais modelos e validação cruzada com os melhores resultados para a aplicação deste modelo. Assim como no caso do $\mathrm{Cd}$, as maiores concentrações estão dispostas no canto central mais à direita do mapa, como pode ser visualizado na Figura 7 (III). O restante da região apresenta valores estimados inferiores a $20 \mathrm{mg} \mathrm{kg}^{-1}$.

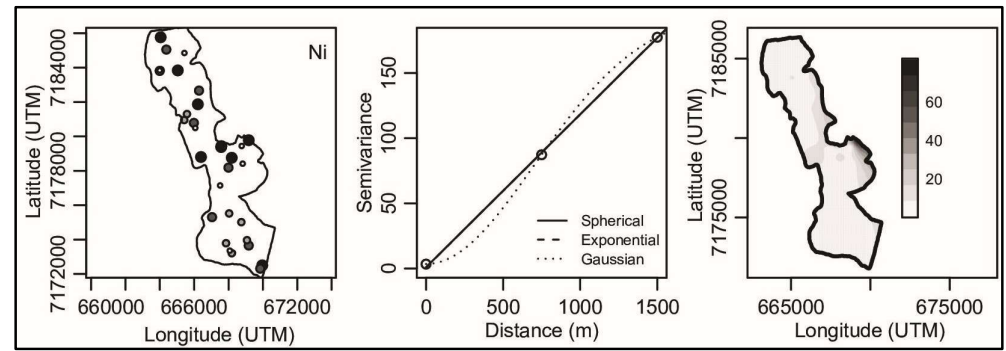

Figura 7: Da esquerda para a direita, os quartis do elemento $\mathrm{Ni}(\mathrm{I})$, semivariograma experimental omnidirecional e modelos ajustados de $\mathrm{Ni}$ (II) e mapas de previsão de distribuição de Ni usando o modelo esférico (III).

É importante destacar que houve um ponto com teor de Ni acima dos valores estabelecidos por Brasil (2009) como "Prevenção" (valor = $30 \mathrm{mg} \mathrm{kg}^{-1}$ ) e "Investigação para área agrícola" (valor $=70 \mathrm{mg} \mathrm{kg}^{-1}$ ). Nesse ponto, sugere-se maior cuidado, pois, além dos valores elevados, o solo deste local é arenoso, atingindo $82 \%$ de areia em sua composição e apenas $7 \%$ de argila.

Nesse caso, o risco de lixiviação é muito grande. Este local está localizado entre uma rua pavimentada e uma vala, em frente a um condomínio industrial. As características do local de amostragem não explicam o valor encontrado, revelando que algum evento inesperado pode ter ocorrido para esse alto valor. A Tabela 1 apresenta uma análise estatística descritiva para a concentração do elemento $\mathrm{Zn}$, com os valores em mg $\mathrm{kg}^{-1}$. O coeficiente de assimetria obtido foi de 0,86 , o menor valor dentre os metais anteriores. Já o valor de curtose foi menor que zero $(-0,21)$, indicando uma curva mais larga que a gaussiana convencional.

O gráfico de quartis (Figura 8 (I)) confirma a existência de pontos próximos com valores similares, dando-nos evidências de que existe dependência espacial. Através dos valores de mínimo $\left(12,32 \mathrm{mg} \mathrm{kg}^{-1}\right)$ e máximo $\left(58,64 \mathrm{mg} \mathrm{kg}^{-1}\right)$. A distância de corte adotada para variograma, onde foi observada correlação especial entre os dados, foi de $380 \mathrm{~m}$. Novamente o modelo esférico foi utilizado para gerar o mapa das estimativas.

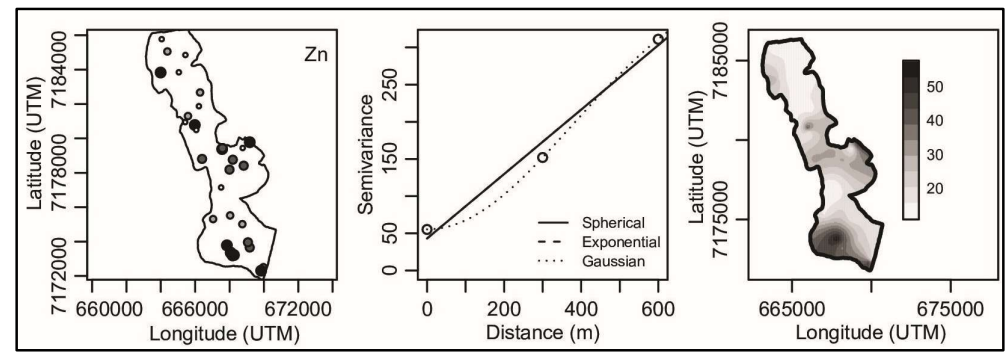

Figura 8: Da esquerda para a direita, os quartis do elemento $\mathrm{Zn}(\mathrm{I})$, semivariograma experimental omnidirecional e modelos ajustados de Zn (II) e mapas de previsão de distribuição de Zn usando o modelo esférico (III).

A Figura 8 (III) refere-se à variação de concentração de Zn estimada para esta região. A coloração clara indica uma menor concentração (abaixo de $20 \mathrm{mg} \mathrm{kg}^{-1}$ ) e a coloração mais escura, próxima ao preto, indica concentrações mais elevadas (próximo a $50 \mathrm{mg} \mathrm{kg}^{-1}$ ). Concomitantemente ao mapa de $\mathrm{Ni}$ e $\mathrm{Cd}$, destacase a região central à direita com os maiores valores de concentração estimada de Zn. Além disso, uma grande 
região inferior do mapa também apresentou valores elevados deste metal.

A avaliação visual dos mapas de incerteza mostra claramente a variação espacial dos elementos-traço na área estudada. As concentrações dos elementos traço estavam abaixo dos valores de prevenção e intervenção da Resolução Brasileira, que é usada como uma legislação de arbitragem no gerenciamento de locais contaminados (BRASIL, 2009).

Tabela 2: Concentração dos metais nas amostras de solo analisadas.

\begin{tabular}{|c|c|c|c|c|c|c|}
\hline Amostras & $\mathrm{Cd}(\%)$ & $\mathrm{Pb}(\%)$ & $\mathrm{Cu}(\%)$ & $\mathrm{Cr}(\%)$ & $\mathrm{Ni}(\%)$ & $\mathrm{Zn}(\%)$ \\
\hline Amostra 1 & 0.26 & 17.45 & 22.54 & 28.49 & 9.17 & 22.09 \\
\hline Amostra 2 & 0.16 & 12.10 & 22.24 & 30.75 & 8.42 & 26.34 \\
\hline Amostra 3 & 0.26 & 13.11 & 12.62 & 20.08 & 5.47 & 48.46 \\
\hline Amostra 4 & 0.17 & 13.09 & 23.24 & 20.11 & 6.26 & 37.14 \\
\hline Amostra 5 & 0.22 & 13.23 & 21.35 & 39.46 & 8.84 & 16.89 \\
\hline Amostra 6 & 0.16 & 17.89 & 23.46 & 27.56 & 7.91 & 23.03 \\
\hline Amostra 7 & 0.16 & 16.52 & 18.32 & 31.00 & 7.39 & 26.61 \\
\hline Amostra 8 & 0.15 & 11.90 & 33.42 & 24.41 & 6.35 & 23.76 \\
\hline Amostra 9 & 0.15 & 9.17 & 22.71 & 36.21 & 11.23 & 20.52 \\
\hline Amostra 10 & 0.28 & 15.67 & 19.75 & 29.36 & 9.15 & 25.80 \\
\hline Amostra 11 & 0.21 & 18.53 & 18.66 & 25.52 & 8.94 & 28.14 \\
\hline Amostra 12 & 0.19 & 15.16 & 15.97 & 17.76 & 6.84 & 44.08 \\
\hline Amostra 13 & 0.21 & 14.70 & 19.79 & 22.83 & 7.16 & 35.32 \\
\hline Amostra 14 & 0.17 & 11.85 & 12.78 & 22.06 & 6.18 & 46.96 \\
\hline Amostra 15 & 0.21 & 8.74 & 19.72 & 42.78 & 10.57 & 17.97 \\
\hline Amostra 16 & 0.12 & 14.59 & 14.00 & 26.33 & 7.13 & 37.83 \\
\hline Amostra 17 & 0.20 & 13.54 & 19.25 & 27.61 & 9.41 & 29.99 \\
\hline Amostra 18 & 0.27 & 18.12 & 21.05 & 31.49 & 7.79 & 21.28 \\
\hline Amostra 19 & 0.20 & 11.45 & 24.66 & 35.48 & 10.87 & 17.33 \\
\hline Amostra 20 & 0.23 & 12.71 & 23.44 & 32.88 & 8.92 & 21.82 \\
\hline Amostra 21 & 0.08 & 14.25 & 23.69 & 33.36 & 13.81 & 14.80 \\
\hline Amostra 22 & 0.16 & 12.70 & 30.55 & 23.35 & 15.04 & 18.20 \\
\hline Amostra 23 & 0.22 & 14.84 & 21.64 & 26.72 & 9.14 & 27.45 \\
\hline Amostra 24 & 0.28 & 8.83 & 15.03 & 23.47 & 5.89 & 46.50 \\
\hline Amostra 25 & 0.24 & 10.95 & 29.03 & 32.79 & 9.18 & 17.81 \\
\hline Amostra 26 & 0.06 & 27.43 & 27.06 & 24.38 & 6.25 & 14.83 \\
\hline Amostra 27 & 0.15 & 13.32 & 19.66 & 24.09 & 14.46 & 28.32 \\
\hline Amostra 28 & 0.28 & 4.35 & 12.94 & 17.59 & 38.38 & 26.46 \\
\hline Amostra 29 & 0.26 & 15.10 & 20.38 & 43.74 & 5.97 & 14.55 \\
\hline Amostra 30 & 0.17 & 31.26 & 15.13 & 14.99 & 6.46 & 31.99 \\
\hline Amostra 31 & 0.22 & 7.01 & 24.92 & 25.56 & 14.73 & 27.57 \\
\hline Amostra 32 & 0.27 & 12.29 & 21.71 & 36.76 & 8.03 & 20.94 \\
\hline Amostra 33 & 0.08 & 9.91 & 20.19 & 45.59 & 10.83 & 13.40 \\
\hline Amostra 34 & 0.34 & 14.15 & 27.76 & 30.06 & 8.22 & 19.47 \\
\hline
\end{tabular}

Embora de acordo com a legislação os valores estejam corretos, comparando todos os mapas obtidos, existe uma tendência de maiores concentrações estimadas na região central do mapa e menores concentrações na região superior. Para verificar a contribuição percentual de cada metal em relação ao conteúdo total, os dados foram normalizados (TOLOSANA-DELGADO et al., 2011). Há predomínio de Cr e Zn nas amostras, atingindo valores de até $48 \%$ para Zn (ver Tabela 2 ).

Embora esses dois metais estejam em proporções mais altas em relação aos outros, eles não representam risco, porque, em comparação com os valores da Resolução Brasileira que é usada como legislação para o gerenciamento de locais contaminados, os valores obtidos estão abaixo de os valores de "Prevenção", que são $75 \mathrm{mg} \mathrm{kg}^{-1}$ para Cr e $300 \mathrm{mg} \mathrm{kg}^{-1}$ para Zn (BRASIL, 2009).

A maior concentração dos elementos- traço pode estar associada a emissões veiculares e indústrias que possam ter contribuído para aumentar o nível desses metais nos solos urbanos analisados. Portanto, 
mesmo a concentração de metais detectados sendo baixa, é importante fazer avaliações da qualidade do solo nas regiões urbanas para permitir o gerenciamento sustentável dos recursos urbanos. Os níveis elevados desses metais podem causar sérias ameaças à saúde da população e do meio ambiente (KUMMER et al., 2011).

\section{CONCLUSÕES}

Nosso estudo demonstra que a análise geoestatística pode fornecer boas estimativas de traços de metais muito valiosos para a avaliação de impacto ambiental. É um excelente veículo de tomada de decisão em relação à qualidade do solo, tanto na verificação quanto no monitoramento ambiental, sem grandes investimentos em amostragem. Tais mapas geoestatísticos permitiram uma melhor visão da dispersão dos traços de metais analisados.

Quanto ao aspecto legal das concentrações de metais pesados nos locais de amostragem, os valores encontrados foram quase todos abaixo dos valores estabelecidos pela legislação brasileira como 'Prevenção', sendo um indicativo da manutenção da qualidade do solo em relação a esses elementos tóxicos, mesmo sendo em uma região tipicamente industrial. A presença de Ni em concentrações superiores à legislação em apenas um ponto da região mostra uma situação pontual e deve ser investigada, principalmente por estar associada a altos níveis de areia, gerando preocupação com a possível contaminação das águas subterrâneas.

AGRADECIMENTOS: Os autores agradecem à Universidade Tecnológica Federal do Paraná (UTFPR) e ao Conselho Nacional de Desenvolvimento Científico e Tecnológico (CNPq) pelo apoio financeiro.

\section{REFERÊNCIAS}

ALMEIDA, S. L. H.; CAPELINI, V. A.; ROSAS J. T. F.; OLIVEIRA, G. D.; LIMA, J. S. S.; SILVA, S. A.. Geoestatística multivariada para a estimativa da temperatura média anual no Estado da Bahia, Brasil. Revista Univap, São José dos Campos, v.22, n.40, 2017. DOI:

http://dx.doi.org/10.18066/revistaunivap.v22i40.1636

BRADY, N. C.; WEIL, R. R.. Elementos da natureza e propriedades dos solos. 3 ed. Porto Alegre: Bookman, 2013.

BRASIL. Resolução CONAMA n. 420, de 28 de janeiro de 2009. Dispõe sobre critérios e valores orientadores de qualidade do solo quanto à presença de substâncias químicas e estabelece diretrizes para o gerenciamento ambiental de áreas contaminadas por essas substâncias em decorrência de atividades antrópicas. Brasília: DOU, 2009.

CHEN, T.; LIU, X.; ZHU, M.; WU, K. Z. J.; XU, J.; HUANG, P.. Identification of trace element sources and associated risk assessment in vegetable soils of the urban-rural transitional area of Hangzhou, China. Environmental Pollution, v.151, p.67-78, 2008. DOI:

http://doi.org/10.1016/i.envpol.2007.03.004

CLARK, I.; HARPER, W. V.. Practical Geostatiscs. Ohio: Ecosse North America LLC, 2000.
COSTA, J.. Caracterização e constituição do solo. Lisboa: Fundação Calouste Gulbenkian, 2004.

CRESSIE, N. A. C.. Statistics for spatial data. Iowa: John Wiley \& Sons, 1993.

CSUROS, M.; CSUROS, C.. Environmental sampling and analysis for metals. Boca Raton: CRC Press, 2002.

CURITIBA. Atividades econômicas, Curitiba, regional CIC (SMF) - 2004 a 2010. Curitiba, 2010.

EMBRAPA. Centro Nacional de Pesquisa de Solos. Manual de métodos de análise de solo. 2 ed. Rio de Janeiro: EMBRAPA, 1997.

FINZGAR, N.; JEZ, E.; VOGLAR, D.; LESTAN, D.. Spatial distribution of metal contamination before and after remediation in the Meza Valley, Slovenia. Geoderma, n.217218, p.135-143, 2014. DOI: http://doi.org/10.1016/j.geoderma.2013.11.011

FREIRE, G.. Caracterização química, física e teores de elementos-traço em solos de diferentes ambientes no Rio Grande do Norte. Dissertação (Mestrado) - Universidade Federal Rural do Semiárido, Natal, 2011 
GARCÍA-LORENZO, M. L.; PÉREZ-SIRVENT, C.; MARTÍNEZSANCHEZ, M. J.; MOLINA-RUIZ, J.. Trace elements contamination in an abandoned mining site in a semiarid zone. Journal of Geochemical Exploration, v.113, p.23-35, 2012. DOI: http://doi.org/10.1016/j.gexplo.2011.07.001

GRUNSKY, E. C.. Statistical analysis in the geosciences, In: ATKINSON, P. M.. Encyclopaedia of Life Support Systems (EOLSS). Oxford: EOLSS Publishers, 2002.

ISAAKS, E. H.; SRIVASTAVA, R. M.. Applied Geostatistics. New York: Oxford University Press, 1989.

IPPUC. Instituto de Pesquisa Planejamento Urbano de Curitiba. A Cidade que queremos: Regional CIC. IPPUC, 2014.

KABATA-PENDIAS, A.. Trace elements in soils and plants. 4 ed. Boca Raton: CRC Press, 2001.

KITANIDIS, P. K.. Introduction to Geostatistics: Applications in Hydrogeology. Cambridge: Cambridge University Press, 1997.

KUMMER, L.; MELO, V. F.; BARROS, Y. J.; AZEVEDO, J. C. R.. Extrações sequenciais de chumbo e zinco em solos de área de mineração e metalurgia de metais pesados. Revista Brasileira de Ciência do Solo, v.35, p.2005-2018, 2011. DOI: http://dx.doi.org/10.1590/S0100-06832011000600017

KUMMER, L.; MELO, V. F.; BARROS, Y. J.. Lead and zinc in the structure of organic and mineral components of soils. Revista Brasileira de Ciência do Solo, v.37, p.438-449, 2013. DOI: http://doi.org/10.1590/S0100-06832013000200015

LADO, L. R.; HENGL, T.; REUTER, H. I.. Heavy metals in European soils: A geostatistical analysis of the FOREGS Geochemical database. Geoderma, v.148, p.189-199, 2008. DOI: http://doi.org/10.1016/j.geoderma.2008.09.020

MARTINS, A. B. T.. Análise geoestatística para dados composicionais. Tese (Doutorado) - Universidade Federal do Paraná, Curitiba, 2010.

MONEGO, M. L. C. D.; RAMOS, P.; NEVES, M. V.. Avaliação de impacto ambiental de descargas de águas residuais usando uma metodologia geoestatística. Revista Brasileira de Recursos Hídricos, v.15, n.3, p.57-68, 2010. DOI: http://dx.doi.org/10.21168/rbrh.v15n3.p57-68

MOURA, R.; FIRKOWSKI, O. L. C. F.. Dinâmicas intrametropolitanas e produção do espaço na Região Metropolitana de Curitiba. Rio de Janeiro: Letra Capital, 2009.

NEZHAD, M. T. K.; MOHAMMADI, K.; GHOLAMI, A.; HANI, A.; SHARIAT, M. S.. Cadmium and mercury in topsoils of Babagorogor watershed, western Iran: Distribution, relationship with soil characteristics and multivariate analysis of contamination sources. Geoderma, v.219-220, p.177-185, 2014. DOI:

http://doi.org/10.1016/i.geoderma.2013.12.021

PEIXOTO, A. P. B.; SANTANA, T. V. F.; HISSA, H. R.; SILVA, E. F.; MACEDO, J. R.; CAPECHE, C. L.; OLIVEIRA, M. C. N.. Análise espacial de dados composicionais. In: RBRAS, 56; SEAGRO, 14. Anais. Maringá, 2011.

PAWLOWSKY-GLAHN, V.; OLEA, R. A.. Geostatistical analysis of compositional data. New York: Oxford University Press, 2004.

R DEVELOPMENT CORE TEAM. R: A Language and environment for statistical computing. Vienna: R Foundation for Statistical Computing, 2016.

RIBEIRO FILHO, J. C.; SANTOS, J. C. N.; ARUAJO NETO, J. R.; LEMOS FILHO, L. C. A.; BRASIL, J. B.. Estimativa das erosividades anuais e mapeamento para o Estado do Ceará. Revista Geonorte, v.8, n.30, p.1-15, 2017. DOI: http://doi.org/10.21170/geonorte.2017.V.8.N.30.1.15

RIBEIRO JUNIOR, P. J.; DIGGLE, P. J.. GeoR: a package for geostatistical analysis. R-NEWS, v.1, p.15-18, 2001.

SABY, N.; ARROUAYS, D.; BOULONNE, L.; JOLIVET, C.; POCHOT, A.. Geostatistical assessment of $\mathrm{Pb}$ in soil around Paris, France. Science of the Total Environment, v.367, p.212-221, 2006. DOI: http://doi.org/10.1016/i.scitotenv.2005.11.028

SANTOS, A. S.; MEDEIROS, N. G.; SANTOS, G. R.; LISBOA FILHO, J.. Uso da Geoestatística na Avaliação da Acurácia Posicional Absoluta de dados Geoespaciais. Boletim de Ciências Geodésicas, v.23, n.3, p.405-418, 2017. DOI: http://doi.org/10.1590/s1982-21702017000300027

SOARES, A.. Geoestatística para as Ciências da Terra e do Ambiente. Coleção Ensino da Ciência e da Tecnologia. Lisboa: IST Press, 2000.

SOBRINHO, N. M. B. A.; CEDDIA, M.B.; ZONTA, E.; MAGALHÃES, M. O. L.; FREITAS, F. C.; LIMA, E. S. A.. Spatial variability and solubility of barium in a petroleum welldrilling waste disposal area. Environmental Monitoring and Assessment, v.190, n.228, p.1-11, 2018. DOI: https//doi.org/10.1007/s10661-018-6566-x

TOLOSANA-DELGADO, R.; OTERO, N.; PAWLOWSKY-GLAHN, V.. Some Basic Concepts of Compositional Geometry. Mathematical Geology, v.37, n.7, p.673-680, 2005. DOI: http://doi.org/10.1007/s11004-005-7374-8

USEPA. United States Environmental Protection Agency. Method 3050B: Acid digestion of sediments, sludges, and soils. USEPA, 1996.

WACKERNAGEL, H.. Multivariate Geostatistics: an introduction with applications. Berlin: Springer Heidelberg, 2003.

A CBPC - Companhia Brasileira de Produção Científica (CNPJ: 11.221.422/0001-03) detém os direitos materiais desta publicação. Os direitos referem-se à publicação do trabalho em qualquer parte do mundo, incluindo os direitos às renovações, expansões e disseminações da contribuição, bem como outros direitos subsidiários. Todos os trabalhos publicados eletronicamente poderão posteriormente ser publicados em coletâneas impressas sob coordenação da Sustenere Publishing, da Companhia Brasileira de Produção Científica e seus parceiros autorizados. Os (as) autores (as) preservam os direitos autorais, mas não têm permissão para a publicação da contribuição em outro meio, impresso ou digital, em português ou em tradução. 